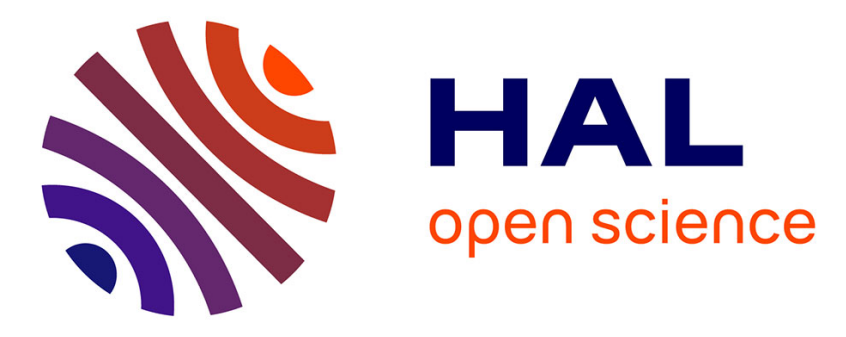

\title{
$\Gamma$-X mixing in type-II GaAs/AlAs short period superlattices
}

Valia Voliotis, R. Grousson, P. Lavallard, E. Ivchenko, A. Kiselev, R. Planel

\section{To cite this version:}

Valia Voliotis, R. Grousson, P. Lavallard, E. Ivchenko, A. Kiselev, et al.. Г-X mixing in type-II GaAs/AlAs short period superlattices. Journal de Physique IV Proceedings, 1993, 03 (C5), pp.C5237-C5-240. 10.1051/jp4:1993545 。 jpa-00251631

\section{HAL Id: jpa-00251631 https://hal.science/jpa-00251631}

Submitted on 1 Jan 1993

HAL is a multi-disciplinary open access archive for the deposit and dissemination of scientific research documents, whether they are published or not. The documents may come from teaching and research institutions in France or abroad, or from public or private research centers.
L'archive ouverte pluridisciplinaire HAL, est destinée au dépôt et à la diffusion de documents scientifiques de niveau recherche, publiés ou non, émanant des établissements d'enseignement et de recherche français ou étrangers, des laboratoires publics ou privés. 


\title{
$\Gamma \cdot X$ mixing in type-II GaAs/AlAs short period superlattices
}

\author{
V. VOLIOTIS, R. GROUSSON, P. LAVALLARD, E.L. IVCHENKO*, A.A. KISELEV* and \\ R. PLANEL**
}

Groupe de Physique des Solides, Universités Paris VI et Paris VII, URA 17 du CNRS, 2 place Jussieu, 75251 Paris cedex 05, France

* A.F. Ioffe Physico-Technical Institute, Russian Academy of Sciences, 194021 St. Petersburg, Russia

** Laboratoire de Microstructures et de Microélectronique, UPR 20 du CNRS, 196 av. Henri Ravera, 92220 Bagneux, France

\begin{abstract}
We have measured the absolute absorption coefficient of optical transitions in type-II short period GaAs/AlAs superlattices, on a broad spectral range, at low temperature. The transmission experiments have been performed in a waveguiding configuration. Photoluminescence excitation experiments show as well the characteristics of the pseudodirect $\mathrm{HH} 1-\mathrm{X}_{\mathrm{z}}$ excitonic transition. Theoretical calculations of the effective dielectric tensor and absorption coefficient in the vicinity of the exciton resonance frequency are presented, taking explicitely into account the $\Gamma-\mathrm{X}$ mixing of electronic states at heteroboundaries. From comparison between the experimental and theoretical values of the absorption coefficients, we have deduced a value of the $\Gamma$-X coupling coefficient for the studied superlattices.
\end{abstract}

\section{INTRODUCTION}

In short period GaAs/AlAs superlattices (SL), a transition from type-I to type-II occurs, when the GaAs layer width is smaller than $35 \AA$ and the mean $\mathrm{Al}$ concentration is greater than $36 \%$ [1]. The carriers are then spatially separated, electrons occupying AlAs X-states, while holes remain confined in GaAs $\Gamma$-wells. For AlAs layer thickness less than $55 \AA$ and GaAs layer thickness larger than $15 \AA$, the lowest confined electron-state is an $\mathrm{X}_{\mathrm{z}}$ state $[1,2]$.

The SL translational symmetry allows mixing between the $\mathrm{X}_{\mathrm{z}}$-valley states and the states lying at or near the $\Gamma$ conduction band edge. The $\Gamma-\mathrm{X}_{2}$ mixing is the origin of the weakly allowed $\mathrm{HH}$ (or LH)$X_{\mathrm{z}}$ optical transitions in type-II SLs. However, their oscillator strength is expected to be reduced as compared to type-I transitions because of the carriers spatial separation. The amount of $\Gamma-X$ mixing has been evaluated with a disparity of values by several groups $[3,4,5,6]$. Some of the authors $[3,5]$ proposed to consider as a mechanism responsible for mixing not only the superperiodicity of the ideal SL but also the random potential due to the thickness fluctuations at the interfaces.

In this paper, we present absolute absorption experiments carried out on optical planar waveguides formed by type-II GaAs/AlAs short period SLs, at low temperature. The absolute absorption coefficient was determined by a study of the luminescence reabsorption [7, 8]. A complete theoretical calculation is performed within the generalized envelope function approximation proposed by Ando and Akera [9] and corrected by Aleiner and Ivchenko [10].

\section{OPTICAL WAVEGUIDING EXPERIMENTS}

The advantages of the waveguiding geometry have been discussed in details in [8]. It is worth noticing that we observe the transmission, on a broad spectral range, in both polarization directions, parallel and normal to the plane of layers and that this geometry allows us to measure absorption coefficients varying from a few $\mathrm{cm}^{-1}$ to several tenths of thousands of $\mathrm{cm}^{-1}$ in the case of a single quantum well $[7,8]$. 


\subsection{Samples}

The samples used in the absorption experiments are optical planar waveguides made of GaAs/AlAs short period SLs grown by MBE at $630^{\circ} \mathrm{C}$ on a (001) oriented GaAs substrate and characterized by X-ray diffraction. Sample 1 is formed by $2.148 \mu \mathrm{m}$ of a (6/4) monolayers GaAs/AlAs short period SL (labelled SL1), grown on a GaAs substrate. A $200 \AA$ GaAs cap layer is grown on top of the sample. This sample is a leaky guide since the refractive index of the GaAs substrate is the highest. The losses in the substrate are of the order of $50 \mathrm{~cm}^{-1}$ for the fundamental mode [8]. The guide is designed to be quasi-monomode.

Sample 2 , grown on a GaAs substrate, is formed by $1 \mu \mathrm{m}$ of a $(2 / 15)$ monolayers GaAs/AlAs SL, $0.4 \mu \mathrm{m}$ of a (4/9) monolayers GaAs/AlAs SL (labelled SL2), $0.5 \mu \mathrm{m}$ of a $(2 / 15)$ monolayers GaAs/A1As SL. A $200 \AA$ GaAs capping layer ends the growth sequence. Sample 2 is designed in such a way that the optical mode propagating along the structure is localized in the SL of interest (SL2). Therefore, this sample is a guide with very weak losses and the absorption experiment gives directly the absorption of the SL itself. It is also a monomode structure. SL1 and SL2 are both type-II SLs. SL1 is close to the type-I to type-II transition [1]. The lowest confined electron-state is an $\mathrm{X}_{\mathrm{z}}$ electron-state in AlAs, lying 30 to $40 \mathrm{meV}$ below the $\Gamma$ electron-state in GaAs. SL2 is also in the $X_{z}$ region. The energy difference between the $X_{z}$ and $\Gamma$ electron states is $\sim 350 \mathrm{meV}$. One expects that the absorption coefficient in SL2 is much weaker than in SL1.

\subsection{Experimental setup}

The experimental setup has been described in details in [8]. The transmission experiments are done with a quartz halogen lamp at low temperature. The coupling between light and sample is obtained by focusing the incident beam on the sample with a microscope objective of great aperture. The transmitted light is collected by a mirror-objective which has also a great aperture. The transmitted intensity is detected through a spectrometer by a photomultiplier, followed by a lock-in amplifier.

The PL experiments are performed at $4 \mathrm{~K}$ with the $514.5 \mathrm{~nm}$ line of a $\mathrm{cw} \mathrm{Ar}{ }^{+}$laser and the PLE spectra are recorded using a tunable $\mathrm{Ar}^{+}$-pumped-dye-laser (Rhodamine-6G).

\subsection{Experimental results}

Fig.1 shows at low temperature the absolute absorption of SL1 in the polarization perpendicular to the growth axis $\mathrm{z}$, the PL and the PLE spectra. One sees clearly the onset of absorption at $1.954 \mathrm{eV}$ followed by a plateau attributed to the excitonic transition $\mathrm{HH} 1-\mathrm{X}_{\mathrm{z}}$, involving electrons in $\mathrm{X}_{\mathrm{z}}$ states in AlAs layers and heavy-holes at $\Gamma$-point in GaAs layers. Let us notice that no excitonic peak is clearly resolved. The steep rise of absorption at higher energy is attributed to the direct HH1- $\Gamma$ transition. The absorption coefficient of the HH1- $\mathrm{X}_{\mathrm{z}}$ excitonic transition on the plateau is measured to be equal to 360 $\mathrm{cm}^{-1}$. This value is by two orders of magnitude weaker than the absorption coefficient in a type-I SL with the same period. A tail of absorption remains on the low-energy side of the absorption curve and is likely due to localized exciton states. The PLE spectrum shows the same features as the absorption experiment.

The PL consists of a main zero-phonon line attributed to the recombination of the $\mathrm{X}_{\mathbf{z}}-\mathrm{HH} 1$ localized excitons, shifted by $11 \mathrm{meV}$ from the absorption line. Two phonon replicas appear at lower energy, at $34 \mathrm{meV}$ and $48 \mathrm{meV}$ with respect to the zero-phonon line.

In Fig. 2 are presented the absorption spectra of SL2 in the parallel and perpendicular polarizations and the PL spectrum. The PL curve shows like in SL1, a main zero-phonon line at $1.989 \mathrm{eV}$ assigned to type-II excitons. On the low energy side of the main line, appear two phonon replicas of the transition, at 1.964 and $1.942 \mathrm{eV}$. The PL zero-phonon line is Stokes shifted from the absorption peak by about $20 \mathrm{meV}$. This shift is explained, as in the case of SL1, by the localization of excitons.

The absorption spectrum in the perpendicular polarization shows a "hump" at $2.01 \mathrm{eV}$, attributed to the $\mathrm{HH} 1-\mathrm{X}_{\mathrm{z}}$ excitonic transition of SL2 (Fig.2). The absorption coefficient is very weak, a few $\mathrm{cm}^{-1}$. On the high energy side of the $\mathrm{HH} 1-\mathrm{X}_{\mathrm{z}}$ transition we observe a quasi-linear variation of the absorption versus energy which extrapolates roughly at $30 \mathrm{meV}$ higher than the exciton peak. This absorption is attributed to indirect absorption processes with phonon emission. This indirect absorption is expected indeed to be a linear function of energy since the 2D-density of states is a constant. The phonons involved in the process are difficult to identify and several can participate to the absorption. Let us note that in the parallel polarization, we observe a linear variation of the absorption versus energy, shifted by $30 \mathrm{meV}$ from the linear curve in the parallel polarization. Since the $X_{x y}$ states lie at 20-30 meV above the $\mathrm{X}_{\mathrm{Z}}$ states, we attribute this absorption to the phonon assisted $\mathrm{HH} 1-\mathrm{X}_{\mathrm{xy}}$ transitions. This unexpected polarization selection rule can be explained if we assume that the electron-phonon interaction connects the 
valence band extrema at $\mathbf{k}=0$ and at the zone boundary. In this case, the optical transition should occur at the zone boundary with modified selection rules.

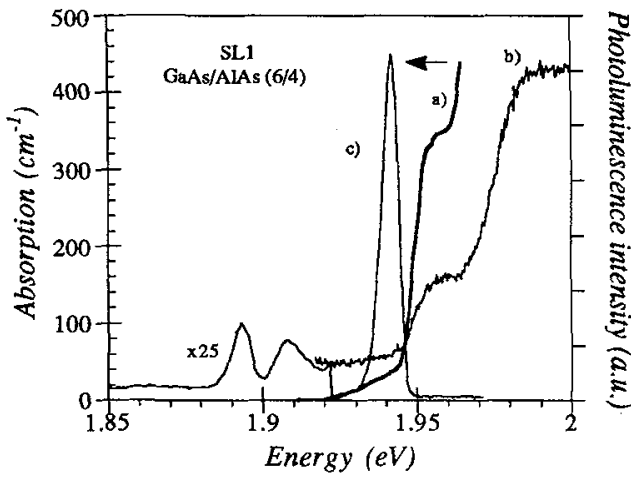

Fig.1. (a) Absorption in the $\mathbf{E} \perp z$ polarization, (b) PLE and (c) PL spectra of SL1 at low temperature. The detection energy was set at $1.894 \mathrm{eV}$ and the scanned energy range was between 1.989 and $2.17 \mathrm{eV}$.

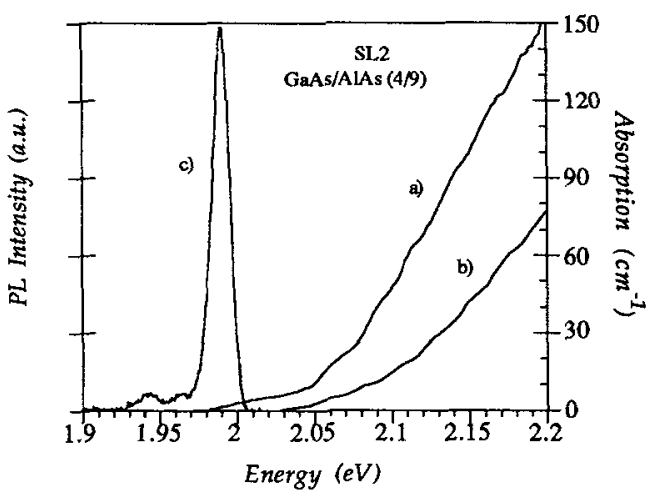

Fig.2. (a) Absorption in the $\mathbf{E} \perp \mathrm{z}$ polarization, (b) in the $E / / z$ polarization and $c$ ) PL spectra of SL2 at low temperature.

\section{THEORY AND DISCUSSION}

In the approximation of an optically-homogeneous effective medium [11], the absorption coefficient $\alpha_{\perp}$ in the $\mathbf{E} \perp z$ polarization, can be represented near the HH1- $X_{z}$ exciton resonance frequency, $\omega_{0}$, as

$$
\alpha_{\perp}(\omega)=\frac{\omega}{c} \sqrt{\varepsilon_{\mathrm{b}}} \frac{\omega_{\mathrm{LT}} \Gamma}{\left(\omega_{\mathrm{o}}-\omega\right)^{2}+\Gamma^{2}}
$$

Here, $\Gamma$ is the exciton damping considered as a phenomenological parameter, $\varepsilon_{\mathrm{b}}$ is the background dielectric constant, $\omega_{\mathrm{LT}}$ is the exciton longitudinal-transverse splitting (or oscillator strength) given by

$$
\omega_{\mathrm{LT}}=\frac{8 \mathrm{e}^{2} \mathrm{p}_{\mathrm{cv}}{ }^{2}}{\hbar \omega_{\mathrm{o}}{ }^{2} \mathrm{~m}_{\mathrm{o}}{ }^{2} \varepsilon_{\mathrm{b}} \mathrm{d} \mathrm{a}_{\perp}{ }^{2}}\left(\int \mathrm{C}_{\mathrm{e}}(\mathrm{z}) \phi_{\mathrm{h}}(\mathrm{z}) \mathrm{dz}\right)^{2},
$$

where $p_{c v}$ is the interband matrix element $i\left\langle S\left|p_{x}\right| X\right\rangle, d$ is the SL period, a trial envelope function describing the relative electron-hole motion is chosen in the exponential form $\left(2 /\left(\pi \mathrm{a}_{\perp}{ }^{2}\right)\right)^{1 / 2} \exp \left(-\rho / \mathrm{a}_{\perp}\right), \rho$ being the in-plane electron-hole distance and $a_{\perp}$ being the effective Bohr radius. We also use in Eq. (2) the following notations: $\phi_{h}(z)$ is the envelope of the heavy-hole wave function, $C_{e}(z)$ is the envelope of the $\Gamma$ state contribution to the wave function of an electron in the lowest subband $\mathrm{e} 1 \mathrm{X}_{\mathrm{z}}$. Here we assume the indirect optical transition $\mathrm{HH} 1-\mathrm{X}_{\mathrm{z}}$ to be allowed due to the $\Gamma-\mathrm{X}_{\mathrm{z}}$ mixing at the heteroboundaries. In the generalized effective mass approximation $[9,10]$ (see also $[12]), C_{\mathrm{e}}(\mathrm{z})$ is determined from the continuity requirement at the interface and the second boundary condition that relates the derivative $\partial \mathrm{C}_{\mathrm{e}} / \partial \mathrm{z}$ to an interface value of the $X_{z}$ envelope function. The dimensionless constant $t_{\Gamma X}$ of the $\Gamma$-X mixing is considered as a free parameter which should be estimated from comparison between the theory and the measured absorption spectra. The calculations of $\omega_{\mathrm{LT}}$ are made (i) in the $2 \mathrm{D}$ limit where $\mathrm{a}_{\perp}$ is taken as the 2D Bohr radius and (ii) in the simple quasi-2D exciton model where $a_{\perp}$ is found using the variational procedure with a one parameter trial function. Taking $t_{\Gamma X}=0.1$ and $\sqrt{\varepsilon_{b}}=3.6$ for both SLs, $\Gamma=5$ and $10 \mathrm{meV}$ for SL1 and SL2 respectively, we find in the 2D limit the following results: $\omega_{\mathrm{LT}}=8 \mathrm{~ns}^{-1}$, $\alpha_{m} \equiv \alpha_{1}\left(\omega_{o}\right)=365 \mathrm{~cm}^{-1}$ and $\omega_{\mathrm{LT}}=12 \mathrm{~ns}^{-1}, \alpha_{\mathrm{m}}=565 \mathrm{~cm}^{-1}$ for SL1 with $(6 / 4)$ and (7/4) monolayers respectively. This is in agreement with the experimental value of $\alpha_{\mathrm{m}}=360 \mathrm{~cm}^{-1}$ obtained for SL1. For the same value of $t_{\Gamma X}$, we also obtain a good agreement for SL2. The theoretical values are $\omega_{\mathrm{LT}}=$ 
$0.2 \mathrm{~ns}^{-1}, \alpha_{\mathrm{m}}=4.25 \mathrm{~cm}^{-1}$ and $\omega_{\mathrm{LT}}=0.07 \mathrm{~ns}^{-1}, \alpha_{\mathrm{m}}=1.75 \mathrm{~cm}^{-1}$ for (4/9) and (4/10) monolayers respectively, while the experimental absorption coefficient is of the order of a few $\mathrm{cm}^{-1}$. In the quasi-2D model, one can fit the data with a larger value, $t_{\Gamma X} \sim 0.2$. Thus, the real value of $t_{\Gamma X}$ lies between 0.1 and 0.2 .

In well defined type-II $(\mathrm{GaAs})_{\mathrm{N}}(\mathrm{AlAs})_{\mathrm{M}} \mathrm{SLs}$, the resonant absorption coefficients are independent on the parity of monolayer numbers $\mathrm{N}, \mathrm{M}$. However, the theory predicts a noticeable M-parity dependence for SLs near the type-I to type-II transition.

We also measured the photoluminescence decay in the two SLs. If we assume that some delay after the excitation pulse, the decay is mainly due to the radiative recombination, then the determination of both the absorption coefficient and the radiative lifetime, allows us to estimate the excitonic localization radius as a few tens $\AA$ for the two studied SLs.

\section{CONCLUSION}

We have measured for the first time the absolute value of the absorption coefficient in type-II $\mathrm{GaAs} / \mathrm{AlAs}$ SLs in an optical waveguiding experiment. We have shown that the mechanism of $\Gamma-X$ mixing of electronic states by perfect interfaces can be successfully used for the description of the measured resonance absorption spectra and we have determined the value of the $\Gamma-X$ coupling coefficient for the studied SLs.

\section{Acknowledgements}

One of the authors (E. L. I.), gratefully acknowledges the Centre National de la Recherche Scientifique for financial support.

\section{References}

[1] Scalbert D., Cernogora J., Benoit à la Guillaume C. , Maaref M., Charfi F. F. and Planel R., Solid State Commun. 70 (1989) 945

[2] Van Kesteren H. W., Cosman E. C., Dawson P., Moore K. J. and Foxon C. T., Phys. Rev. B 39 (1989) 13426

[3] Meynadier M. H., Nahory R. E., Worlock J. M., Tamargo M. C., de Miguel J. L. and Sturge M. D., Phys. Rev. Lett. 60 (1988) 1338

[4] Pulsford N. J., Nicholas R. J., Dawson P., Moore K. J., Duggan G. and Foxon C. T., Phys. Rev. Lett. 63 (1989) 2284

[5] Dawson P., Moore K. J., Foxon C. T., W. 't Hooft G. and van Hal R. P. M., J. Appl. Phys. 65 (1989) 3606

[6] Maaref M., Charfi F. F., Scalbert D., Benoit à la Guillaume C., and Planel R., Solid State Commun. 81 (1992) 35

[7] Grousson R., Lavallard P., Voliotis V., Roblin M.L. and Planel R., Proc. Int. Conf. on Optics of Excitons in Confined Systems Italy 1991, IOP Conf. Ser. No 123 (1992) pp.207-210

[8] Grousson R., Voliotis V., Lavallard P., Roblin M.L. and Planel R., Semicond. Sci. Technol.(to be published)

[9] Ando T. and Akera H., Phys. Rev. B 40 (1989) 11609

[10] Aleiner I. A. and Ivchenko E. L., Fiz. Techn. Poluprovodn. 27 (1993) 594 (to be translated in Sov. Phys. Semicond.)

[11] Ivchenko E. L., Sov. Phys. Solid State 33 (1991) 1344

[12] Fu Y., Willander M., Ivchenko E. L., Kiselev A. A., Phys. Rev. B 47 (1993) 13498 